\title{
La red y la aplicación de las nuevas tecnologías en la investigación y aprendizaje de las artes visuales.
}

\section{Paula Santiago Martín de Madrid}

Doctora en Bellas Artes. Profesora de la Facultad de Bellas Artes de la Universitat Politècnica de València. Investigadora en el Centro de Investigación Arte y Entorno CIAE-UPV (masanma6@pin.upv.es)

\begin{abstract}
This paper addresses different activities and teaching methodologies that incorporate the active use of TICS and have been carried out in the context of the course Presentation and disclosure of artwork currently taught in Grade by School of Fine Arts of the Universitat Politècnica de València. Reference is also made to the new virtual spaces that have emerged in recent years in relation to the exhibition of visual arts, what has necessarily affected the adaptation of educational content as well as the design and incorporation practices and activities that help students to develop skills associated with the use of new technologies of communication and information.
\end{abstract}

Keywords: art, exhibition, disclosure, education, university

\begin{abstract}
Resumen
El presente texto aborda diferentes actuaciones y metodologías docentes que han incorporado el uso activo de TICS y que han sido llevadas a cabo en el marco de la asignatura Presentación y divulgación de la obra de arte impartida actualmente en Grado por la Facultad de Bellas Artes de la Universitat Politècnica de València. Asimismo se hace alusión a los nuevos espacios virtuales que han surgido en los últimos años en relación a la exposición de las artes visuales, lo que necesariamente ha incidido en la adaptación de contenidos educativos, así como en el diseño e incorporación de prácticas y actividades que ayuden a la capacitación del alumnado a desarrollar conocimientos asociados al uso de nuevas tecnologías de la comunicación y la información.
\end{abstract}

Palabras clave: arte, exposición, divulgación, educación, universidad 
La red y la aplicación de las nuevas tecnologías en la investigación y aprendizaje de las artes visuales

\section{Introducción}

Actualmente, la creciente configuración de espacios virtuales para la presentación y divulgación de la obra artística -revistas electrónicas, páginas web de museos y centros artísticos, blogs, galerías virtuales, etc.-, han supuesto la incorporación de nuevos contenidos y metodologías docentes encaminadas a potenciar capacidades en nuestros discentes que respondan a las necesidades de este nuevo contexto. En este sentido, partiendo del caso concreto de una de las asignaturas de las que somos responsables docentes, deseamos utilizar la misma con el objetivo de ilustrar la aplicación de determinadas metodologías didácticas que han incorporado el uso de nuevas tecnologías en una doble vertiente. En primer lugar, la relativa al diseño de actividades en cuya metodología se ha incorporado el uso por parte del alumnado de plataformas y recursos externos al ámbito universitario. En segundo lugar, nos centraremos en actuaciones docentes concretas relacionadas con la elaboración de materiales que han sido llevadas a cabo mediante el uso de TICS facilitadas por la institución universitaria.

\section{Metodologías activas y nuevas tecnologías en la asignatura Presentación y divulgación de la obra de arte}

En el marco de la asignatura Presentación y divulgación de la obra de arte se ha adaptado el formato y la temática de la oferta de conocimientos a las nuevas necesidades relacionadas con conocimientos vinculados con la postproducción de la obra de arte, con su divulgación y con las nuevas metodologías de exposición. Este hecho ha motivado algunas de las actuaciones concretas llevadas a cabo, tanto en lo que respecta a la planificación, como al desarrollo de la materia propia de la asignatura. Por tanto, el logro de los objetivos fijados en el ámbito de la asignatura ha requerido el oportuno planteamiento de una serie de actividades definidas por su operatividad y/o funcionalidad real.

Durante los últimos cursos han sido numerosas las prácticas propuestas que han incorporado el manejo de plataformas tecnológicas especializadas. En este sentido, cabe destacar la realización de prácticas reales que han supuesto, entre otras, las siguientes actuaciones por parte del alumnado:

- Búsquedas en la web en los procesos de investigación.

- Búsquedas en la web de convocatorias públicas de carácter artístico en las que $a$ posteriori se ha participado activamente.

(c)) EY-NC-ND 2015, Universitat Politècnica de València

Congreso IN-RED (2015) 
- Incorporación de trabajos creativos y dossiers de obra en plataformas reales especializadas en la difusión y divulgación de las artes.

- Manejo de plataformas para la elaboración de mapas conceptuales en la realización de propuestas de carácter teórico.

- Presentaciones y defensas orales con la elaboración de presentaciones en power point.

- Manejo de plataformas para la organización personalizada de páginas web.

- Elaboración de blogs personalizados de carácter artístico.

A su vez, las cinco metodologías que se detallan a continuación han sido utilizadas en diversos momentos del proceso de enseñanza-aprendizaje. En todos los casos el manejo de nuevas tecnologías ha sido básico para el desarrollo de las mismas.

- Elaboración de proyectos. La elaboración de proyectos supone una metodología muy extendida en la dinámica educativa de Bellas Artes. Esta práctica permite al alumnado desarrollar, tanto en lo que se refiere a la organización como a la aplicación, los conceptos aprendidos. Al mismo tiempo, la estrategia proyectual familiariza con la consulta de fuentes, la búsqueda de información y la redacción de textos, es decir, con todos aquellos procesos vinculados con la investigación. Asimismo, la exposición de los resultados en el contexto del aula ayuda al alumnado a desarrollar capacidades asociadas con la comunicación oral. Para la realización de estos proyectos se ha requerido del alumnado un importante trabajo de búsqueda de información especializada en la web, así como la elaboración de presentaciones de calidad mediante el uso de nuevas tecnologías o la utilización de foros y blogs.

- Estudio de casos. El planteamiento de un problema que no tiene una solución única o absoluta también constituye una práctica habitual en el desarrollo de cuestiones relacionadas con aspectos creativos. El objetivo de esta metodología va encaminada a suscitar el análisis y la reflexión individual para posteriormente poner en común los resultados obtenidos dentro del contexto del aula. Esta dinámica provoca la participación activa de todos los estudiantes, motivándolos e implicándolos en el ejercicio a través de encuestas y foros, así como mediante la utilización de blogs.

- Juego de rol. El hecho de que el alumnado asuma diferentes roles con la intención de simular una vivencia que nos permita comprender el porqué de las situaciones y actitudes de los demás, supone una práctica de gran ayuda. La representación requiere toda una serie de posiciones intercambiables que suponen, entre otras cuestiones, adoptar el rol de experto en una materia concreta, utilizar una terminología propia del papel asumido, desarrollar una mirada crítica, etc. El método requiere actividades de 
La red y la aplicación de las nuevas tecnologías en la investigación y aprendizaje de las artes visuales

juicio crítico en las que el alumnado realiza un trabajo previo de investigación mediante la consulta en páginas especializadas -páginas web de museos, galerías, revistas especializadas, etc.-.

- Foro/Debate dirigido. La discusión en la que intervienen todos los integrantes del grupo mediante la libre expresión de ideas se ha llevado a cabo, por lo general, a continuación de otras actividades como talleres, seminarios o conferencias, así como en otras situaciones en las que se ha partido de un texto y/o noticia de actualidad. Este tipo de práctica ha requerido para su correcto y eficaz desarrollo la combinación de medios tecnológicos con la intervención en el aula.

- El trabajo de campo. Este tipo de prácticas constituye un elemento básico para que el alumnado se familiarice con la realidad profesional. Los objetivos prioritarios han estado encaminados a aproximar al alumno/a a la realidad profesional, no sólo desde una perspectiva creativa, sino también desde una vertiente de gestión. Asimismo, se ha intentado favorecer el conocimiento de las entidades públicas y privadas asociadas a la práctica artística y cultural. En numerosas actividades, el alumnado se ha enfrentado a la necesidad de llevar a cabo simulaciones virtuales adaptadas a espacios reales.

\section{Docencia en un entorno virtual}

En el transcurso de los últimos años se ha propiciado un cambio de paradigma en el entorno educativo, tanto para el alumnado como para el profesorado, que viene determinado por el uso de las nuevas tecnologías. En este sentido cabe destacar un conjunto de iniciativas llevadas a cabo desde la Universitat Politécnica de València con el objetivo de mejorar el proceso enseñanza-aprendizaje:

- En 2005 comenzamos a trabajar con la plataforma PoliformaT, un sistema unificado para la gestión del aprendizaje y el trabajo colaborativo.

- En 2007 y con el objetivo de enriquecer ese entorno de aprendizaje, se aprobó el Plan Docencia en Red destinado a la producción de materiales docentes que pueden consultarse en el Aula Abierta de la Web de la Universitat Politècnica de València.

- En 2008 la UPV se adhirió al Consorcio OpenCourseWare (OCW), una iniciativa que emergió para promover asignaturas on line de acceso libre.

- Recientemente, nuestra institución se ha unido a los MOOC (Masive On line Open Course), movimiento iniciado por la Universidad de Stanford. 
Asimismo, en el marco de la asignatura Presentación y divulgación de la obra de arte, se han aplicado las citadas actuaciones que han supuesto sustituir métodos y técnicas pedagógicas de cara a programar, implementar y evaluar acciones formativas on line. En este epígrafe se hace alusión a una serie de materiales docentes elaborados durante los últimos cursos para cuya elaboración nos hemos ayudado de las tecnologías señaladas y cuya finalidad ha sido la de facilitar el proceso de enseñanza-aprendizaje de nuestro alumnado mediante el uso de las TICS.

\subsection{Un blog para la asignatura}

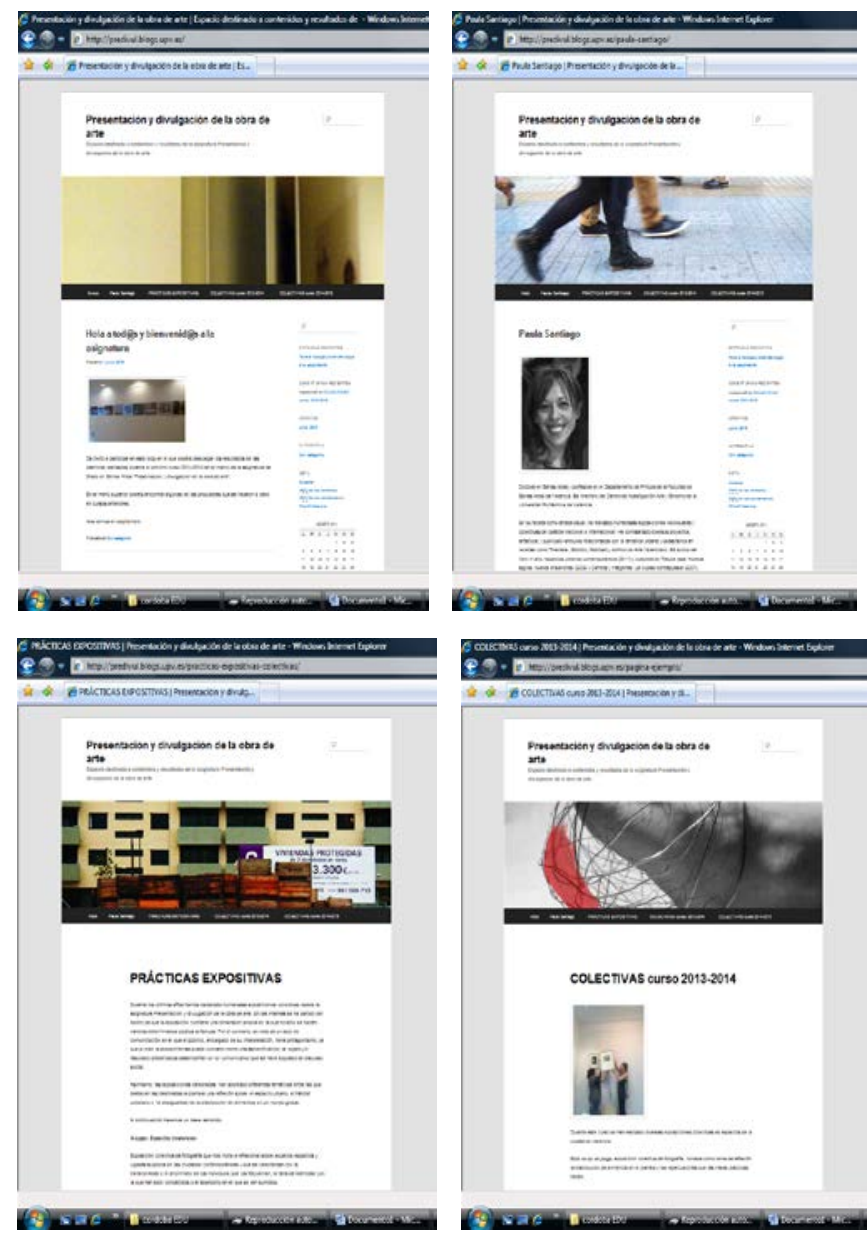

Fig. 1. Paula Santiago. Blog Presentación y divulgación de la obra de arte.

\section{(c)) EY-NC-ND 2015, Universitat Politècnica de València}


La red y la aplicación de las nuevas tecnologías en la investigación y aprendizaje de las artes visuales

Con el objetivo de favorecer el uso de las nuevas tecnologías, así como la participación y motivación del alumnado, hemos generado un blog (fig. 1) específico que recoge impresiones, comentarios, resultados de investigación, materialización de proyectos, etc. derivados de las actividades propuestas en la asignatura. Al mismo tiempo, el citado blog ha servido como plataforma de divulgación de los resultados obtenidos, así como ejemplo para la elaboración de blogs por parte del alumnado. De hecho, una de las actividades incorporadas recientemente en la programación de la asignatura tiene como objetivo la elaboración y puesta en marcha de un blog mediante el cual es alumnado pueda difundir sus proyectos.

\subsection{Polimedias}

Por otro lado, en el marco de la asignatura, hemos recurrido a Polimedia, sistema de producción de materiales educativos de la Universitat Politècnica de València para la creación de contenidos multimedia como apoyo a la docencia presencial, que abarca desde la preparación del material docente hasta su distribución a través de distintos medios tecnológicos: TV, Internet, CD, etc. Haciendo uso de esta herramienta didáctica, hemos publicado durante los últimos cursos 29 vídeos (fig. 2) con diferentes contenidos que han recibido, desde su producción, más de 20.000 visualizaciones en el portal de la UPV y más de 25.000 en YouTube.
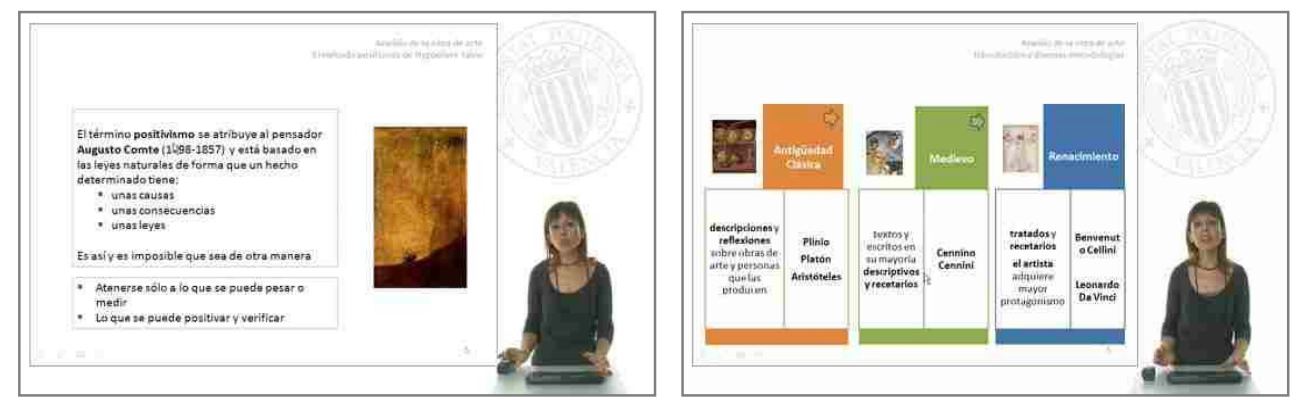

Fig. 2. Paula Santiago. Polimedias educativos.

Su incorporación en los contenidos didácticos de la asignatura ha supuesto un cambio de paradigma en la presentación de materiales. Los mismos han sido objeto de exposiciñon y debate en el aula, razón por la que las clases magistrales han incrementado el índice de participación del alumnado. Asimismo, los objetos de aprendizaje elaborados se han incorporado en el marco de la asignatura como un recurso imprescindible en aquellos casos

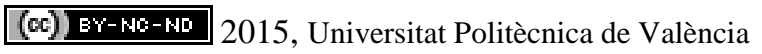

Congreso IN-RED (2015) 
en los que el alumnado necesita de un refuerzo extraordinario para el aprendizaje, facilitando el repaso de contenidos.

\subsection{Asignatura $\mathrm{OCW}$}

En la misma dirección, los contenidos y materiales elaborados para la asignatura han sido publicados en el portal OCW (figura 3) incluyendo un conjunto significativo de materiales que, a su vez, son de libre acceso a través de internet. Los materiales han abarcado: actividades formativas, contenidos de teoría de aula, prácticas de aula, etc.

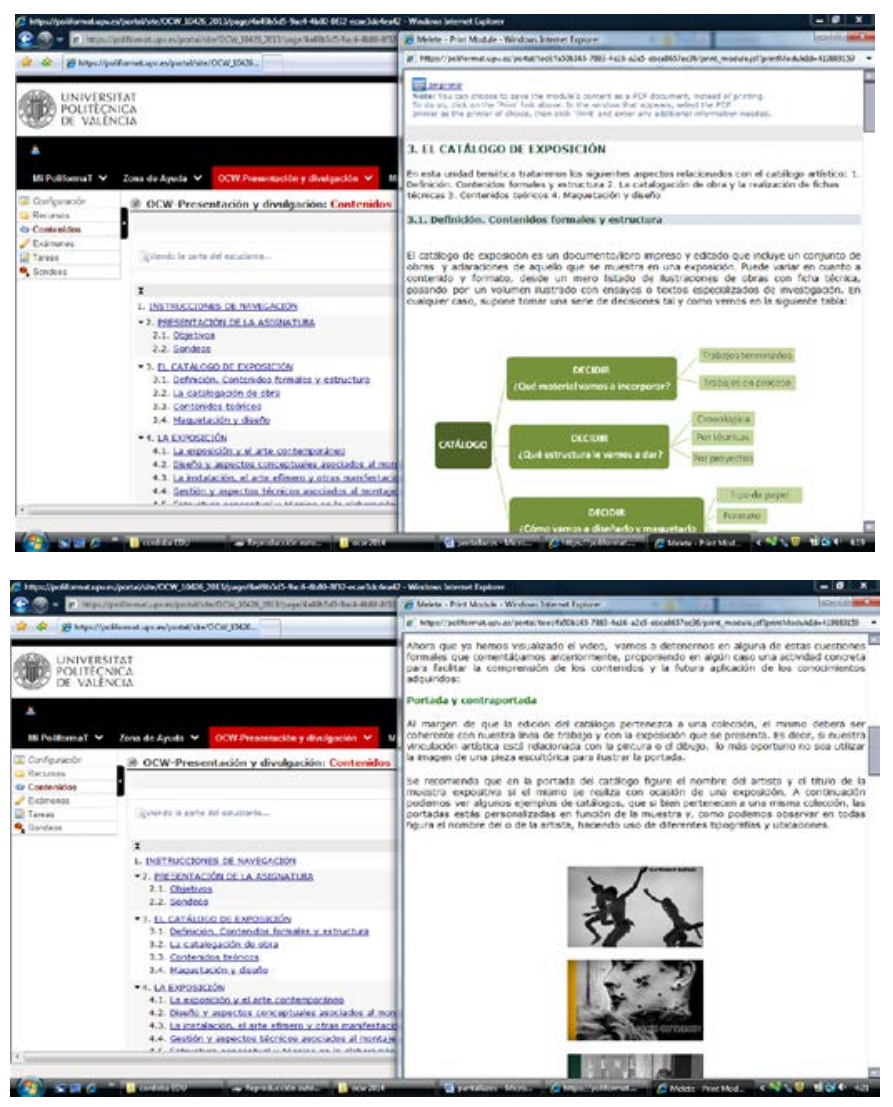

Fig. 3. Paula Santiago. Asignatura OCW.

\section{(c)) EY-NC-ND 2015, Universitat Politècnica de València}


La red y la aplicación de las nuevas tecnologías en la investigación y aprendizaje de las artes visuales

\subsection{PoliformaT}

La Plataforma PoliformaT ha sido utilizada permanentemente en la práctica docente de la asignatura. Todos los materiales usados han sido publicados en la misma: programa de la asignatura, bibliografía, criterios de evaluación, cronograma, guía docente, presentaciones utilizadas en las clases magistrales, fichas descriptivas de las actividades propuestas, ejemplos y anexos de las citadas actividades, así como otros materiales y propuestas puntuales. A su vez, otras aplicaciones de esta plataforma (correo electrónico, espacios compartidos, tablón de anuncios, foros, calendario, etc.) han sido de utilización cotidiana, tanto para el profesorado como para el alumnado en la práctica docente de la asignatura.

\section{Conclusiones}

El objetivo del presente comunicado ha sido el de exponer una serie de actuaciones docentes llevadas a cabo durante los últimos cursos con el objetivo de adaptar el formato a las nuevas necesidades relacionadas con conocimientos vinculados con la postproducción de la obra de arte, con su divulgación y con las nuevas metodologías de exposición. Para ello se ha requerido del planteamiento de una serie de actividades cuyos objetivos, en relación al uso de nuevas tecnologías, se pueden resumir en los siguientes:

1. Fomentar el uso de metodologías activas que incorporan el uso de nuevas tecnologías.

2. Plantear prácticas en contextos reales que faciliten el conocimiento de nuevas plataformas en la presentación y divulgación de la obra de arte.

3. Favorecer el uso de la red en los procesos de investigación y difusión.

4. Adaptar diferentes metodologías docentes al nuevo contexto virtual.

De los resultados obtenidos y en relación con el discente, podemos destacar que:

- El alumnado ha accedido de forma activa a plataformas incipientes en la difusión de la obra de arte, familiarizándose con las nuevas dinámicas, fomentando sus relaciones más allá del marco universitario.

- Se ha potenciado el desarrollo tanto de las capacidades específicas propias de la materia como de las transversales.

- El uso de las nuevas tecnologías y de la red en las prácticas a desarrollar ha favorecido la motivación del alumnado así como la dinámica de aula.

Desde una perspectiva de acción docente:

(c)) EY-NC-ND 2015, Universitat Politècnica de València

Congreso IN-RED (2015) 
- Las actuaciones que han incorporado el uso de TICs y que hemos citado en el presente texto, han requerido de la aplicación y adaptación de métodos y técnicas pedagógicas concretas, tanto en la programación y diseño de actividades como en la evaluación de las diferentes acciones formativas on line.

- Estimando los resultados obtenidos, podemos decir que el proceso de enseñanzaaprendizaje de nuestro alumnado se ha visto favorecido notablemente, con una mayor participación y motivación. 